\title{
Short versus long biliopancreatic limb in Roux-en-Y gastric bypass surgery for treatment of type 2 diabetes mellitus
}

\author{
Zhigang Keㄹ, Fan $\mathrm{Li}^{1}$, Yu Gao${ }^{1}$, Xunmei Zhou ${ }^{2}$, Fang Sun², Li Wang ${ }^{1}$, Jing Chen², Xin Tan ${ }^{3}$, Zhiming Zhu², Weidong Tong ${ }^{1}$ \\ ${ }^{1}$ Department of General Surgery, Daping Hospital, Army Medical University, Chongqing, China \\ ${ }^{2}$ Department of Hypertension and Endocrinology, Daping Hospital, Army Medical University, Center for Hypertension and Metabolic \\ Diseases, Chongqing Institute of Hypertension, Chongqing, China \\ ${ }^{3}$ Department of Rehabilitation Medicine, Chongqing Bishan People's Hospital, Chongqing, China
}

Videosurgery Miniinv 2021; 16 (1): 129-138

DOI: https://doi.org/10.5114/wiitm.2020.99997

\begin{abstract}
Introduction: Although laparoscopic Roux-en-Y gastric bypass (RYGB) is still widely accepted as a valid procedure in the treatment of obesity and type 2 diabetes mellitus (T2DM), there continues to be a significant controversy about how long the Roux and biliopancreatic limb should be bypassed for optimum results.

Aim: To assess the effect of a longer biliopancreatic limb (BPL) length on glycemic control after RYGB in T2DM patients. Material and methods: Eighty-four patients with uncontrolled T2DM who underwent RYGB between May 2010 and April 2017 were collected from the prospectively designed database. Forty patients (S-BPL group) received BPL lengths $\leq 50 \mathrm{~cm}$, including $30 \mathrm{~cm}(n=1), 40 \mathrm{~cm}(n=1)$, and $50 \mathrm{~cm}(n=38)$. Forty-four patients (L-BPL group) received $100 \mathrm{~cm}$ BPL. Anthropometry, serum glucose and lipid metabolic parameters were measured at baseline and 1, 3, 6, 12,24 and 36 months after surgery.

Results: Comparing the two groups, there were no significant differences in anthropometric and biochemical measures, except the weight and body mass index, which were higher in the S-BPL group $(85.91 \pm 20.32$ vs. $76.25 \pm 16.99$, $p=0.038 ; 31.87 \pm 6.61$ vs. $28.7 \pm 4.29, p=0.005)$ compared to the L-BPL group. The body weight, glucose and lipid metabolic parameters decreased over time and then remained essentially stable from the first year in both groups. Two years after surgery, the remission $\left(\mathrm{HbA}_{1 c} \% \leq 6 \%\right)$ of T2DM was $31.2 \%$ in the S-BPL group and $37.5 \%$ in the L-BPL group $(p=0.685)$.

Conclusions: With consistent total small bowel bypass $(A L+B P L)$ lengths, lengthening of the $B P L$ from 30 to $100 \mathrm{~cm}$ did not affect the post-RYGB glycemic control and weight loss.
\end{abstract}

Key words: Roux-en-Y gastric bypass, type 2 diabetes mellitus, biliopancreatic limb.

\section{Introduction}

The Roux-en-Y gastric bypass (RYGB) is currently still the most preferable surgical intervention to treat morbid obesity and type 2 diabetes mellitus (T2DM). The total number of bariatric procedures was approximately 54490 from 31 countries in the 3 calendar years $2013-2015$, of which $49.4 \%$ were RYGB procedures [1]. But still, there is no consensus on the ideal gastric bypass limb lengths. Reported lengths of the biliopancreatic limb (BPL) and alimentary limb (AL) varied widely from $10-250$ to $35-250 \mathrm{~cm}$, respectively, in a survey of 215 American bariatric surgeons by the American Society for Bariatric Surgery [2].

A number of comparative studies have been performed with variable lengths of the AL, and they show controversy in the effect on weight loss

\section{Address for correspondence}

Weidong Tong PhD, Department of General Surgery, Daping Hospital, Army Medical University, Chongqing 400042, China,

e-mail: vdtong@163.com 
[3-16]. A few studies have revealed the relationship between the lengths of the bypassed limb and the remission of T2DM. Pinheiro et al. [12] reported that patients with longer AL and BPL achieved greater type 2 diabetes control and lipid disorder improvement. Kaska et al. [17] found that a longer BPL can intensify the anti-diabetic effect of RYGB. However, with the extension of $A L$ and BPL length, the common limb (CL) becomes shorter. Many authors also have demonstrated that weight loss and remission of T2DM after RYGB are strongly associated with a short common limb. Unfortunately, the shorter the common limb, the more malnutrition and metabolic complications can be expected $[4,18,19]$. Therefore, how to choose the length of the bypass limb has a vital impact on balancing the efficacy of surgery and nutritional complications in RYGB.

\section{Aim}

Generally, an AL length of 100 to $150 \mathrm{~cm}$ and a BPL length of 25 to $100 \mathrm{~cm}$ with an unmeasured CL length were recommended $[2,20]$. In this study, we kept the total bypass $(A L+B P L)$ lengths consistent

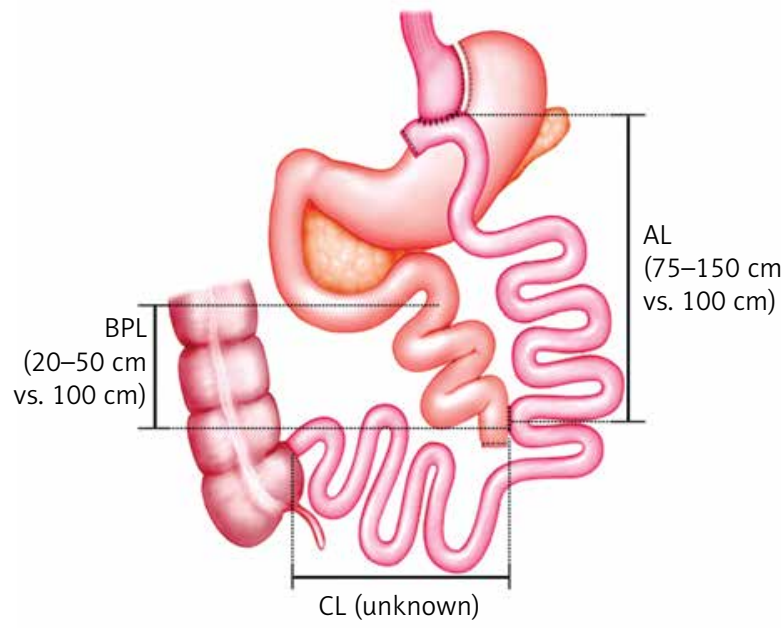

Figure 1. Schematic drawing of operation and limb lengths: In standard BPL group patients, the length of the biliopancreatic limb (distance from the ligament of Treitz to the jejunojejunostomy) is $20-50 \mathrm{~cm}$ and the alimentary limb (distance from the gastrojejunostomy to the jejunojejunostomy) is $75-150 \mathrm{~cm}$. In longer BPL group patients, the length of the biliopancreatic limb is $100 \mathrm{~cm}$ and the alimentary limb is $100 \mathrm{~cm}$

$B P L$ - biliopancreatic limb, $A L$ - alimentary limb, $C L$ - common limb length. in two groups, and extended the BPL length in order to evaluate its clinical efficacy in T2DM remission.

\section{Material and methods}

\section{Patient selection and follow-up}

Eighty-four patients with uncontrolled T2DM who underwent RYGB at our hospital from May 2010 to April 2017 were retrieved from a prospectively designed database. The protocol for this study was approved by the Ethics Committee and institutional review at our hospitals and was compliant with the Helsinki Declaration.

The inclusion criteria were as follows: diagnosis of T2DM or other important co-morbidity based on the criteria of the American Diabetes Association (ADA) [21], from 18 to 60 years of age, body mass index $(\mathrm{BMI})>25 \mathrm{~kg} / \mathrm{m}^{2}$ (based on Chinese obesity and type 2 diabetes surgical treatment guidelines (2014)). A patient would be excluded if he or she had previously undergone bariatric surgery or other complex abdominal surgery, as were those with established diagnoses of type 1 diabetes, latent adult autoimmune diabetes, malignancy, pregnancy, neurologic disease or cardiovascular disease.

Prior to the operation, each patient was assessed by a multidisciplinary team (MDT) comprising a surgeon, endocrinologist, anesthetist, psychiatrist and dietician. Moreover, each patient underwent a routine preoperative workup and counseling in addition to a detailed diabetic workup. Patients were followed up at the outpatient endocrinology clinic with weight registration and metabolic (glucose and lipid) surveillance. The date sets of baseline and 1, 3, 6, and 12 months after surgery and annually thereafter were collected.

\section{Surgical procedure}

Laparoscopic RYGB was performed by a single surgeon; the technical aspects of the procedures were the same. For LRYGB, five trocars were used, constructing a $25-30 \mathrm{ml}$ gastric pouch, the gastrojejunostomy was created by a staple technique with an anastomosis $1.5-2.0 \mathrm{~cm}$ in diameter, and the integrity of the anastomosis was tested with methylene blue solution infused through an orogastric tube. The length of the biliopancreatic limb was $20-50 \mathrm{~cm}$ in the short biliopancreatic limb (S-BPL) group and $100 \mathrm{~cm}$ in the long biliopancreatic limb (L-BPL) group, 
with alimentary limb values of 75-150 $\mathrm{cm}$ and $100 \mathrm{~cm}$ in S-BPL and L-BPL respectively (Figure 1). The mesenteric and Petersen defects were closed.

\section{Outcomes}

The primary end point was the proportion of patients with a glycated hemoglobin $\left(\mathrm{HbA}_{1 c}\right)$ level of $6 \%$ or less (with or without diabetes medications) for at least 12 months [22]. Secondary end points included levels of fasting plasma glucose (FPG), 2-hour postprandial glucose (2hPG), fasting C-peptide, $\mathrm{HbA}_{1 \mathrm{c}}$, serum total cholesterol (TC), triglyceride (TG), high-density lipoprotein (HDL), low-density lipoprotein (LDL), arterial blood pressure, BMI and waist circumference.

\section{Statistical analysis}

Statistical analysis was performed using SPSS version 17.0 (SPSS Inc, Chicago, IL, USA). Graphs were made using a commercially available software package (GraphPad Prism for Windows). Baseline comparisons were performed using the $\chi^{2}$ test, paired $t$ test, and one-way ANOVA. Continuous variables were expressed as mean \pm standard deviation, continuous variables were compared using Student ANOVA for repeated measurement, and a two-tailed $p$-value $<0.05$ was considered statistically significant.

\section{Results}

\section{Patients}

We retrospectively evaluated the results of $84 \mathrm{pa}$ tients with T2DM who underwent laparoscopic RYGB, including 38 (45.2\%) women and 46 (54.8\%) men. They were divided into two groups based on the length of BPL. Forty patients were enrolled in the $\mathrm{S}-\mathrm{BPL}$ group, including $30 \mathrm{~cm}(n=1), 40 \mathrm{~cm}(n=1)$, and $50 \mathrm{~cm}(n=38)$. The L-BPL group consisted of 44 patients who received $100-\mathrm{cm}$ limbs. The number of follow-up patients was $51(60.7 \%)$ at 1 year, $40(47.6 \%)$ at 2 years, $13(15.5 \%)$ at 3 years. The baseline patient characteristics are shown in Table I. Comparing the two groups, there were no significant differences in age, duration of diabetes, blood pressure, or glucolipid metabolism. However, the patients of the S-BPL group had significantly greater weight $(85.91 \pm 20.32$ vs. $76.25 \pm 16.99 \mathrm{~kg}$, $p=0.038)$, higher BMI (31.87 \pm 6.61 vs. $28.7 \pm 4.29 \mathrm{~kg} / \mathrm{m}^{2}$, $p=0.005)$ and higher C-peptide level (2.01 \pm 2 vs. $1.08 \pm 0.66 \mathrm{ng} / \mathrm{ml}, p=0.01$ ).

\section{Complications}

All procedures were successfully performed by laparoscopic techniques. There was no early or late mortality. Eight (9.5\%) patients developed early or late complications, including 1 patient with gastrojejunal anastomotic leakage, 2 patients with gastrojejunal anastomotic stenosis, 2 patients with anastomotic ulcer, 2 patients with anemia and 1 patient with severe malnutrition. All such complications were cured through conservative treatment.

\section{Glycemic control}

In both groups, the FPG, $2 \mathrm{hPG}, \mathrm{HbA}_{1 \mathrm{c}} \%$ and C-peptide levels steadily decreased in the first 6 months $(p<0.05)$, and remained stable during follow-up (Figure 2). Although a trend of better glucose metabolic control was observed in the L-BPL group, there was no significant difference at any point between the S-BPL and L-BPL groups (Figures $2 \mathrm{~A}-\mathrm{C}$ ). In 1-year follow-up after surgery, $\mathrm{C}$-peptide in the S-BPL group was higher than that of the L-BPL group ( $p<0.05)$ (Figure $2 \mathrm{D}$ ).

The remission of T2DM at one year is shown in Table II. Eleven (52.3\%) patients in the S-BPL group and 17 (56.7\%) patients in the L-BPL group achieved complete remission of diabetes mellitus with $\mathrm{HbA}_{1 c}$ $<6.0 \%(p=0.762)$, and $18(85.7 \%)$ patients in the S-BPL group and 26 (86.7\%) patients in the L-BPL group achieving improvement of diabetes mellitus with $\mathrm{HbA}_{1 c}<7.0 \%(p=0.923)$.

The remission of T2DM at 2 years is shown in Table III. Five (31.2\%) patients in the S-BPL group and 9 (37.5\%) patients in the L-BPL group achieved complete remission of diabetes mellitus with $\mathrm{HbA}_{1 \mathrm{c}}$ $<6.0 \%(p=0.685)$, and $13(81.2 \%)$ patients in the S-BPL group and 26 (79.2\%) patients in the L-BPL group achieved improvement of diabetes mellitus with $\mathrm{HbA}_{1 \mathrm{c}}<7.0 \%(p=0.872)$.

\section{Weight loss}

Patients in both groups showed a significant mean weight, BMI and waist circumference reduction. Three years after surgery, the mean weight had declined from $85.91 \pm 20.32$ to $59.36 \pm 10.94(p=$ $0.007)$, BMI had declined from $31.87 \pm 6.61$ to 23.23 $\pm 2.02(p=0.01)$, and waist circumference had decreased from $102.21 \pm 12.65$ to $80 \pm 6.55$ ( $p<0.001)$ in 
Table I. Baseline patient characteristics

\begin{tabular}{|c|c|c|c|}
\hline Characteristic & BPL group $(n=40)$ & BPL group $(n=44)$ & $P$-value \\
\hline Male/female & $23 / 17$ & $23 / 21$ & 0.631 \\
\hline Age [years] & $42.7 \pm 11.7$ & $44.77 \pm 9.4$ & 0.065 \\
\hline Duration of diabetes [years] & $3.13 \pm 3.55$ & $6.01 \pm 4.22$ & 0.152 \\
\hline Weight [kg] & $85.91 \pm 20.32$ & $76.25 \pm 16.99$ & $0.038^{*}$ \\
\hline BMI [kg/m²] & $31.87 \pm 6.61$ & $28.7 \pm 4.29$ & $0.005^{\star}$ \\
\hline Waist circumference [cm] & $102.21 \pm 12.65$ & $95.85 \pm 11.14$ & 0.099 \\
\hline $\mathrm{SBP}[\mathrm{mm} \mathrm{Hg}]$ & $132.38 \pm 15.7$ & $131.86 \pm 15.5$ & 0.841 \\
\hline $\mathrm{DBP}[\mathrm{mm} \mathrm{Hg}]$ & $81.15 \pm 11.3$ & $80.8 \pm 11$ & 0.976 \\
\hline $\mathrm{FPG}[\mathrm{mmol} / \mathrm{l}]$ & $8.12 \pm 3.31$ & $8.59 \pm 3.07$ & 0.93 \\
\hline $2 \mathrm{hPG}[\mathrm{mmol} / \mathrm{l}]$ & $14.53 \pm 5.12$ & $17.1 \pm 4.27$ & 0.321 \\
\hline $\mathrm{HbA}_{1 \mathrm{c}}(\%)$ & $8.01 \pm 2.31$ & $8.35 \pm 1.77$ & 0.169 \\
\hline C-peptide [ng/ml] & $2.01 \pm 2$ & $1.08 \pm 0.66$ & $0.01^{\star}$ \\
\hline $\mathrm{TC}[\mathrm{mmol} / \mathrm{l}]$ & $4.65 \pm 1.1$ & $5.18 \pm 1.37$ & 0.51 \\
\hline $\mathrm{TG}[\mathrm{mmol} / \mathrm{l}]$ & $2.73 \pm 2.21$ & $2.96 \pm 2.49$ & 0.867 \\
\hline $\mathrm{HDL}[\mathrm{mmol} / \mathrm{l}]$ & $1.17 \pm 0.81$ & $1.26 \pm 0.96$ & 0.552 \\
\hline LDL [mmol/l] & $2.5 \pm 0.61$ & $2.97 \pm 0.91$ & 0.194 \\
\hline
\end{tabular}

$B M I$ - body mass index, SBP-systolic blood pressure, DBP - diastolic blood pressure, FPG - fasting plasma glucose, 2hPG - 2-hour postprandial blood glucose, $H b A_{1 c}$ - glycated hemoglobin $A_{1 c}$, TC - total cholesterol, TG - triglyceride, HDL-high-density lipoprotein, LDL - low-density lipoprotein, ${ }^{*} p<0.05$.

the S-BPL group. In the L-BPL group, the mean weight decreased significantly from $76.25 \pm 16.99$ to 55.65 \pm 6.48 ( $p<0.001)$, BMI decreased from $28.7 \pm 4.29$ to $22.21 \pm 1.33(p<0.001)$, and $\mathrm{HbA}_{1 \mathrm{c}}$ decreased from $95.85 \pm 11.14$ to $81.42 \pm 6.6(p<0.001)$. The changes in weight, BMI and waist circumferences among the subjects in both groups are shown in Figures $3 \mathrm{~A}-\mathrm{C}$. The changes in weight, BMI and waist circumference were not different at any point between the S-BPL and L-BPL groups, except in the 1-month post-surgery follow-up, when $\mathrm{BMI}$ was higher in the S-BPL group than the L-BPL group $(p<0.05)$.

\section{Lipid levels and blood pressure}

Post-operative serum total cholesterol (TC) and triglyceride (TG) showed a sharp fall in the first month in both groups, recovered slightly at 1 year and remained stable in the following years (Figures $4 \mathrm{~A}, \mathrm{~B}$ ). The serum HDL and LDL cholesterol remained stable in the follow-up period (Figures $4 \mathrm{C}$, D). The serum levels of TC, TG, HDL, and LDL were similar at each time point for both groups $(p>0.05)$.

The systolic and diastolic blood pressure decreased from baseline to 3 years in both groups, albeit not significantly (Figures $5 \mathrm{~A}, \mathrm{~B}$ ), and there were no differences at any point between groups $(p>0.05)$.

\section{Discussion}

RYGB is considered to be a restrictive and malabsorptive procedure, creating a small gastric pouch (usually 15-30 ml) which limits the amount of food that can be ingested, and bypassing of the duodenum and part of the small bowel provides a degree of malabsorption, together resulting in weight loss and glycemic control. Particularly, the "foregut and hindgut hypotheses" described by Rubino and Cummings explain the hormonal and metabolic benefits of bypassing the duodenum and part of the proximal jejunum and accelerating the undigested food to the distal intestine [23-25], which plays an important role in the treatment of T2DM.

Over the past decades, researchers have sought to achieve greater weight loss by reducing the pouch size and lengthening the limb length. A number of studies have focused on the effect of the AL length and weight loss [26, 27], while less research has been performed to explore the effect of various BPL on 
A

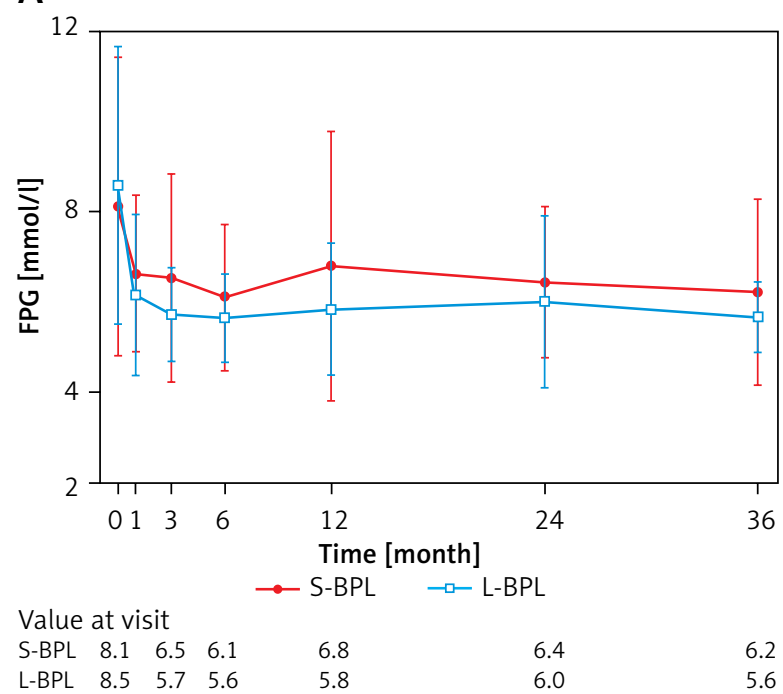

C

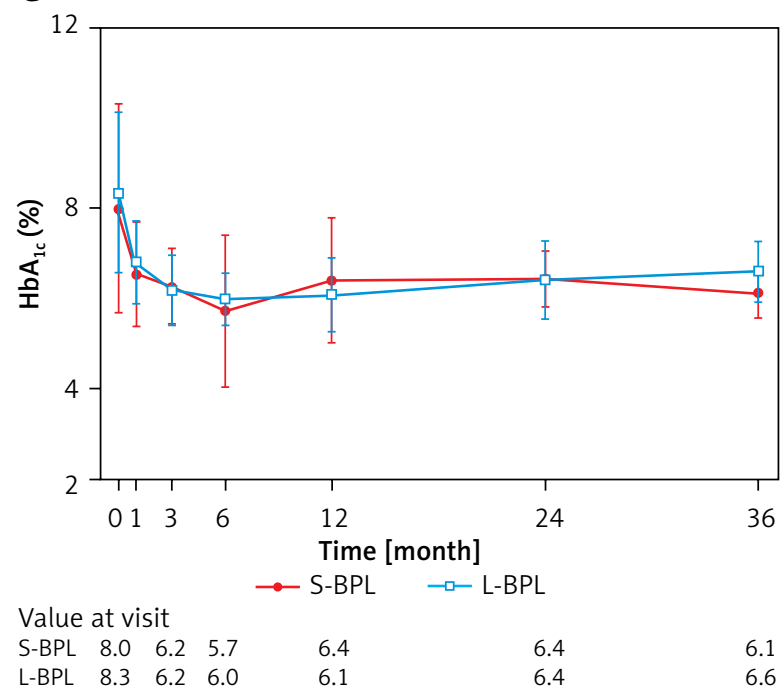

B

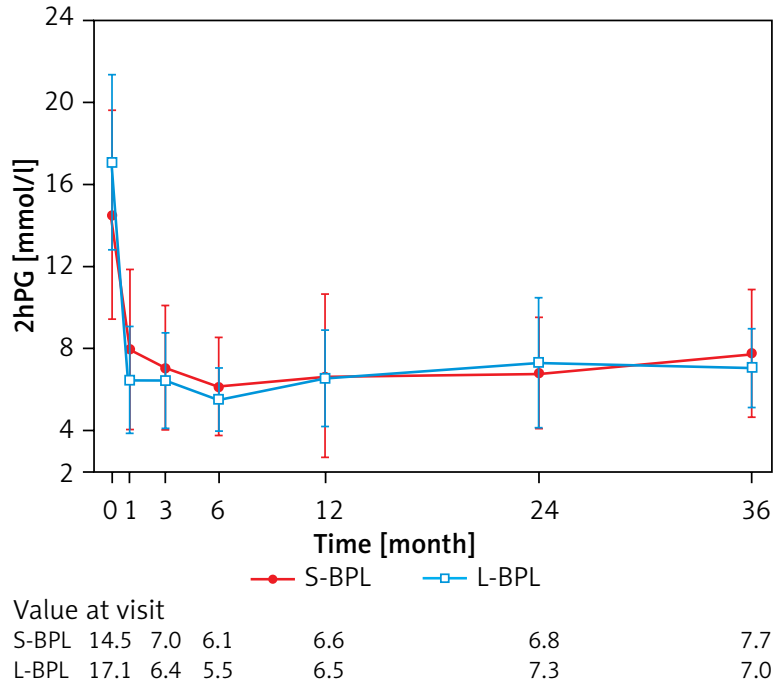

D

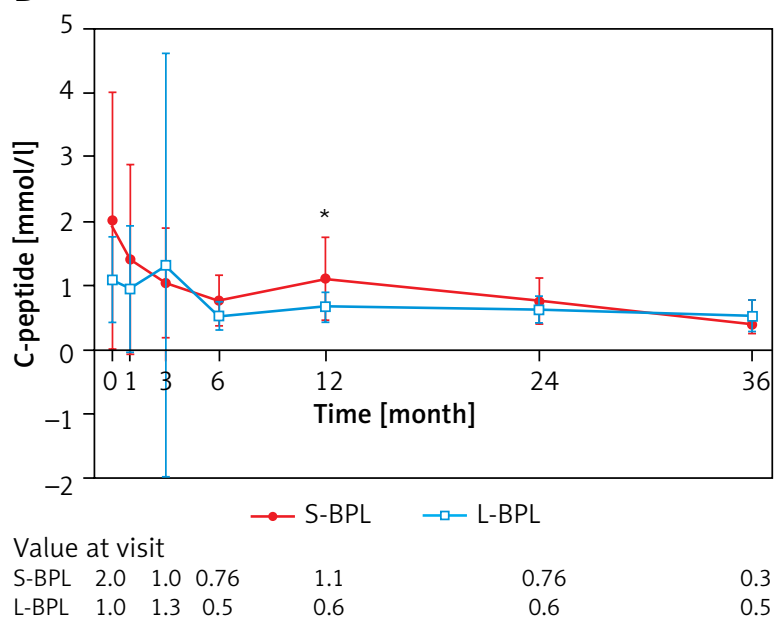

Figure 2. Mean changes in measures of diabetes control from baseline to 3 years between the two groups. A - Fasting plasma glucose (FPG). B - 2-hour postprandial blood glucose (2hPG). C - Glycated hemoglobin $A_{1 c}$ $\left(\mathrm{HbA}_{1 \mathrm{c}}\right)$. D - C-peptide

${ }^{*} p<0.05$.

Table II. Remission of type 2 diabetes mellitus in group of S-BPL and L-BPL at 1 year

\begin{tabular}{|lccc|}
\hline Glycated hemoglobin & S-BPL $(n=21)$ & L-BPL $(n=30)$ & $P$-value \\
\hline$\leq 6.0 \%, n(\%)$ & $11(52.3)$ & $17(56.7)$ & 0.762 \\
\hline$\leq 6.5 \%, n(\%)$ & $13(61.9)$ & $25(83.3)$ & 0.084 \\
\hline$\leq 7.0 \%, n(\%)$ & $18(85.7)$ & $26(86.7)$ & 0.923 \\
\hline
\end{tabular}

S-BPL - short biliopancreatic limb, L-BPL - long biliopancreatic limb. 
Table III. Remission of type 2 diabetes mellitus in group of S-BPL and L-BPL at 2 years

\begin{tabular}{|lccc|}
\hline Glycated hemoglobin & S-BPL $(n=16)$ & L-BPL $(n=24)$ & $P$-value \\
\hline$\leq 6.0 \%, n(\%)$ & $5(31.2)$ & $9(37.5)$ & 0.685 \\
\hline$\leq 6.5 \%, n(\%)$ & $8(50)$ & $15(62.5)$ & 0.433 \\
\hline$\leq 7.0 \%, n(\%)$ & $13(81.2)$ & $19(79.2)$ & 0.872 \\
\hline
\end{tabular}

S-BPL - short biliopancreatic limb, L-BPL - long biliopancreatic limb.

weight loss and T2DM remission $[12,17]$. In the study by Dallegrave Marchesini [28] and Stefanidis et al. [29], a better anti-diabetic effect and a higher T2DM remission rate were demonstrated in patients with a longer BPL, not with a longer AL. An editorial comment indicated that manipulating BP limb length may hold a key in optimizing the effect of RYGB [30].

A

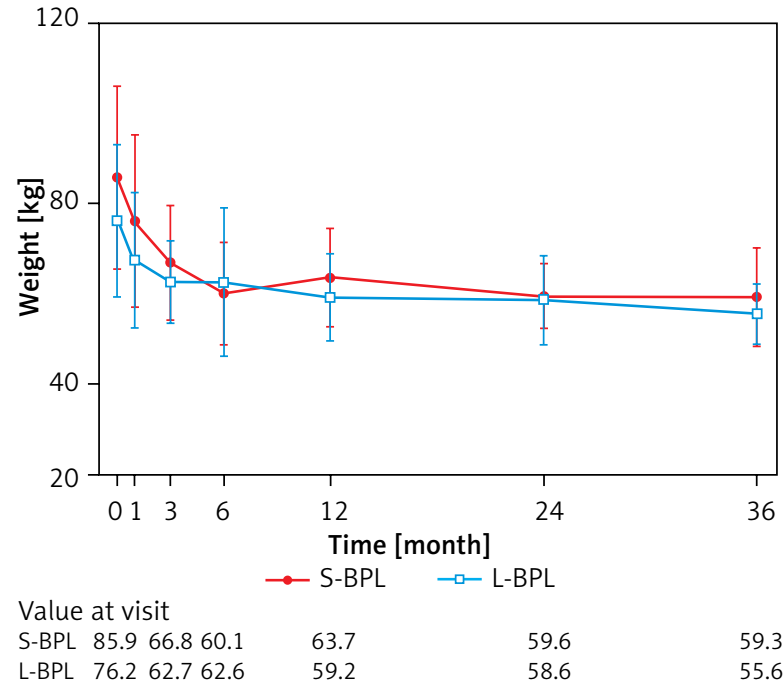

C

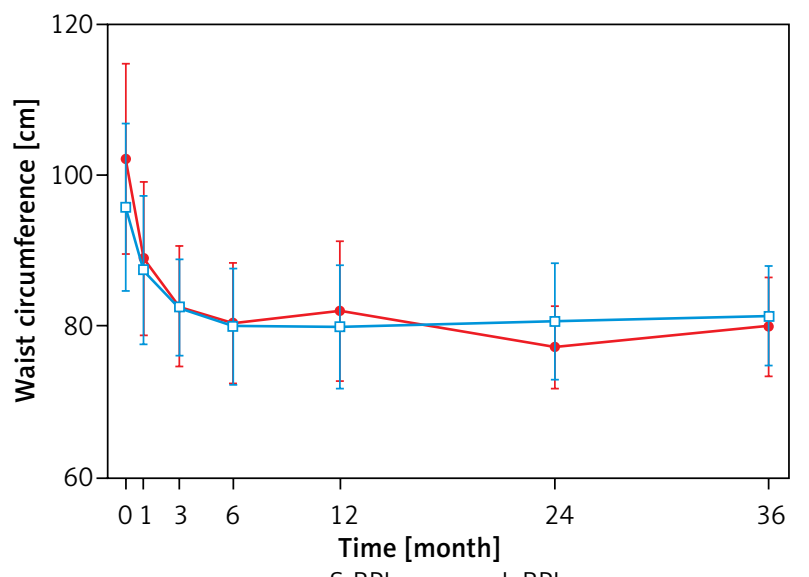

Value at visit

S-BPL 102.282 .780 .5

$\rightarrow$ S-BPL $\quad-$ - L-BPL

L-BPL $95.882580 .0 \quad 80.0$

80.0
There is a case reported by Kao et al. [31], a woman (BMI: $34.4 \mathrm{~kg} / \mathrm{m}^{2}$ ) with T2DM, who had undergone RYGB three times. For the first RYGB, BPL was set at $150 \mathrm{~cm}$, and $A L$ was $100 \mathrm{~cm}$, after 18 months, her BMI and $\mathrm{HbA}_{1 \mathrm{c}}$ were $25.2 \mathrm{~kg} / \mathrm{m}^{2}$ and $4.6 \%$, but frequent diarrhea occurred and medical treatment for this was unsuccessful. So she underwent a laparoscopic

B

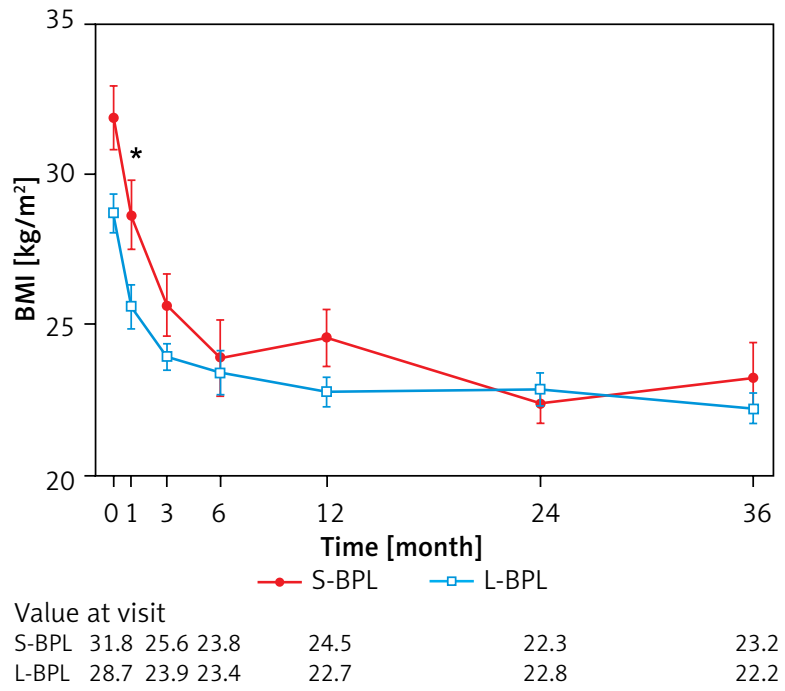

Figure 3. Changes in weight (A), body mass in$\operatorname{dex}(B)$ and waist circumference (C) from baseline to 3 years between the two groups ${ }^{*} p<0.05$. 
A

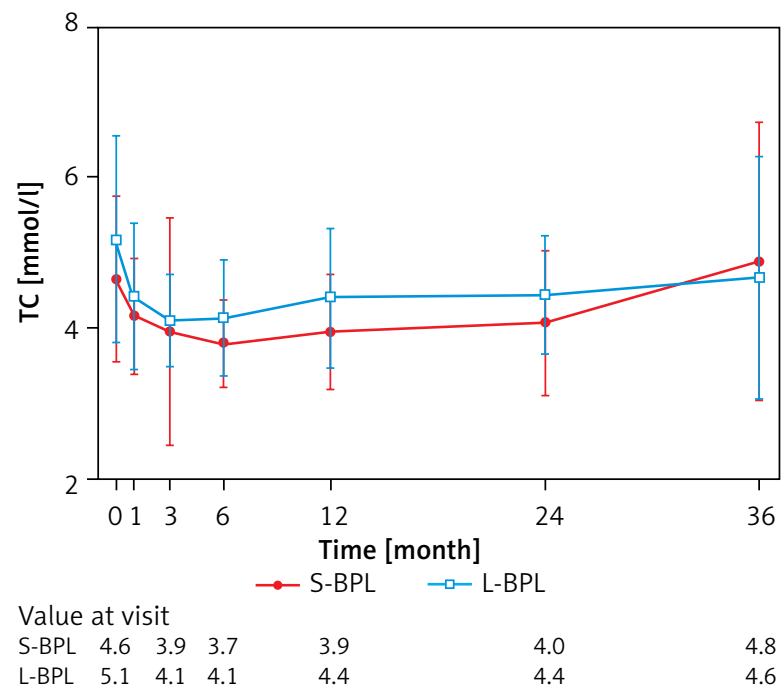

C

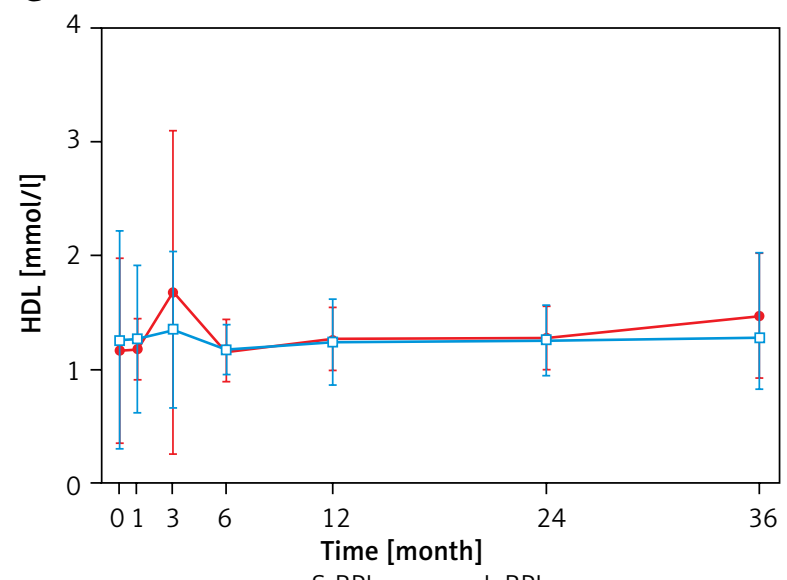

Value at visit

$\begin{array}{lllll}\text { S-BPL } & 1.1 & 1.6 & 1.1 & 1.2\end{array}$

$\rightarrow$ S-BPL $\rightarrow-$ L-BPL
B

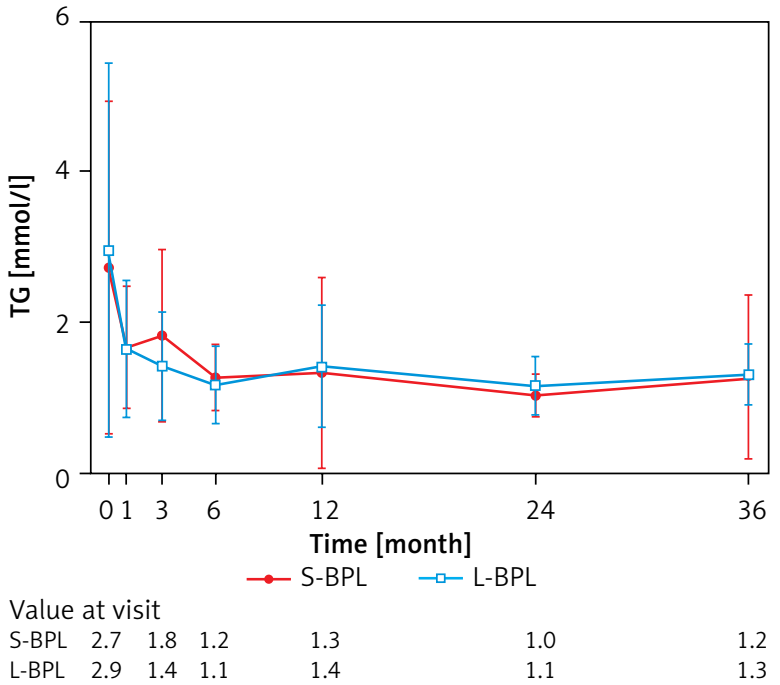

D

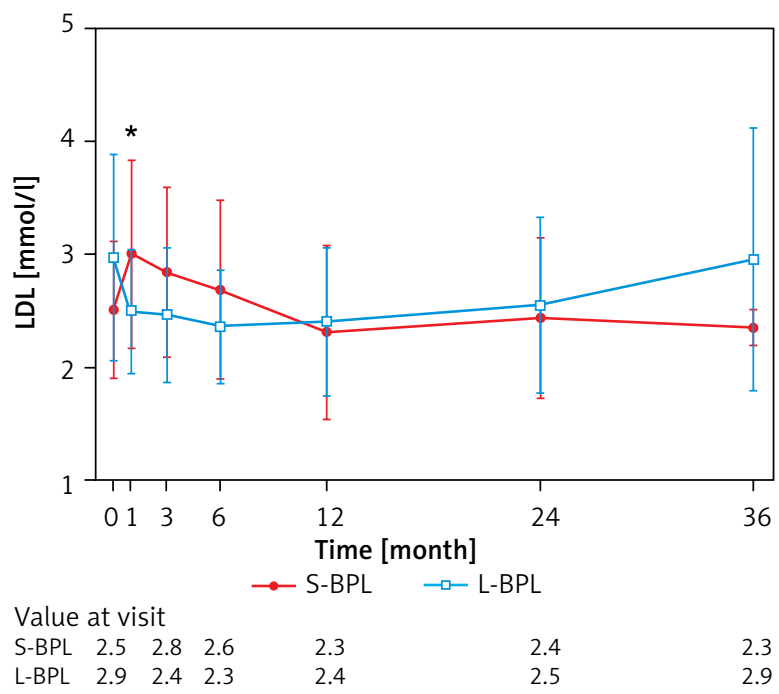

Figure 4. Mean changes in measures of lipid profile from baseline to 3 years between the two groups. A - Total cholesterol (TC). B - Triglycerides (TG). C - High-density lipoprotein (HDL). D - Low-density lipoprotein (LDL)

revision, bypass limbs were shortened (BPL: $40 \mathrm{~cm}$, $A L: 70 \mathrm{~cm}$ ), her diarrhea improved, but T2DM recurred immediately after the reoperation. Subsequently, a second revision procedure was performed again, during which the bypassed limbs were lengthened (BPL: $100 \mathrm{~cm}, \mathrm{AL}: 70 \mathrm{~cm}$ ). Eventually, T2DM remission was achieved again after the last operation. Therefore, the BPL length plays a key role in the treatment of type 2 diabetes after RYGB.

Also, some previous studies have proved that the degree of malabsorption after gastric bypass is influenced mainly by the length of the $C L$ rather than the lengths of the AL or BPL [29]. The better long-term weight loss and type 2 diabetes control described by some studies when longer $\mathrm{AL}$ and $\mathrm{BPL}$ are used are likely a reflection of the shortening common channel $[12,32]$, but the shorter the common limb, the more malnutrition and metabolic complications can be expected $[18,19]$. Unfortunately, few studies have taken into consideration the length of the common channel, so we have a limited understanding of how long the common channel should be to achieve the best weight loss and glucose control outcomes without increasing the incidence of nutritional complica- 
A

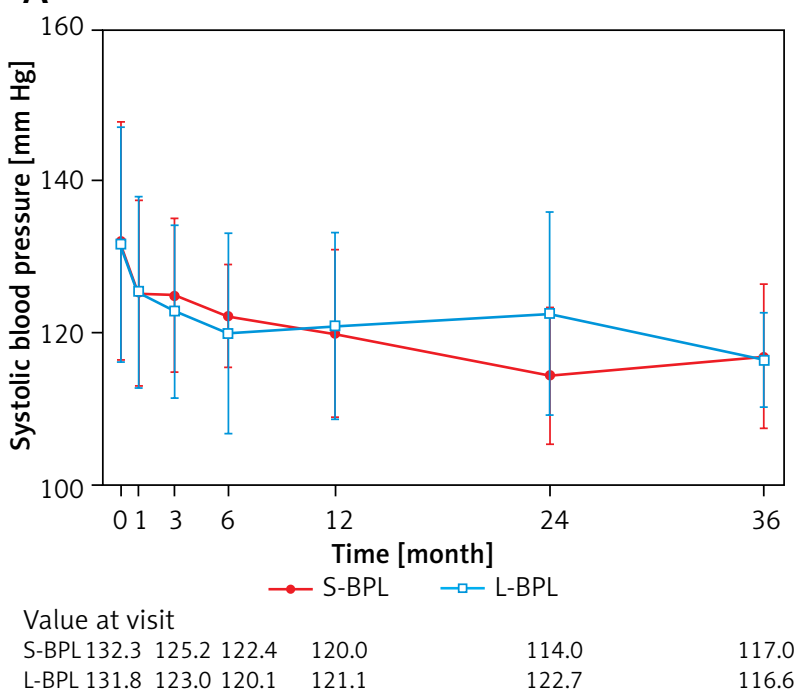

B

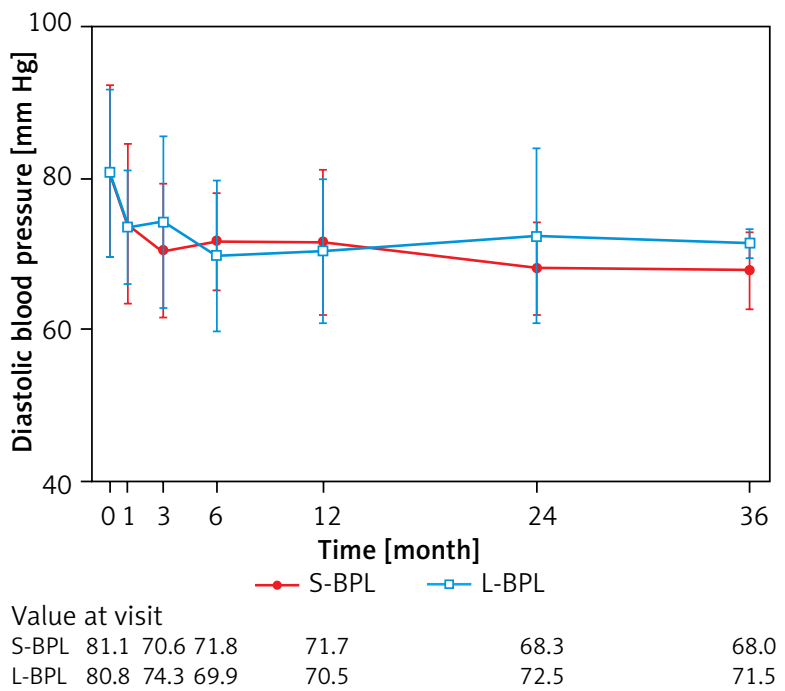

Figure 5. Changes in blood pressure from baseline to 3 years between the two groups. A - Systolic blood pressure, $\mathbf{B}$ - diastolic blood pressure

tions. Many studies have shown that the total small bowel length can be extremely variable; a range of 300 to $900 \mathrm{~cm}$ is a widely accepted figure [27]. Therefore, we have to consider the total small bowel length in RYGB, given that the three limb lengths ( $A L$, $B P L$ and $C L$ ) are interdependent; the longer $A L$ and/ or $\mathrm{BPL}$ are, the shorter the $\mathrm{CL}$ becomes.

In this study, the length of total bypass $(A L+B P L)$ was set at $200 \mathrm{~cm}$ in most patients, with unknown length of the common limb, and a few patients in the early stages of the operation with a short bypassed limb (range: 100-150 cm), so that we have kept long enough $\mathrm{CL}$ to reduce the risk related to the high degree of malabsorption from too short common limb. We wondered whether extending the BPL can improve clinical efficacy in T2DM remission. In a recent evidence-based review, Mahawar et al. came to the conclusion that RYGB achieves optimum results when the combined length of $B P L$ or $A L$ is between 100 and $200 \mathrm{~cm}$. Furthermore, small differences in the proportion of BPL and AL do not result in significantly different outcomes. Our research also verified the conclusion. In our study, there was a trend of more reduction in the glucose metabolism and weight loss for the L-BPL group, but there was no significant difference at any point in the S-BPL and L-BPL groups. So future studies should examine greater differences in the lengths of $B P L$ and $A L$.

The improvements of diabetes, hypertension and dyslipidemia play important roles in reducing microvascular disease, cardiovascular complications and all-cause mortality. In T2DM patients, lipid abnormalities are thought to be secondary to obesity, insulin resistance, and decreased insulin production [33]. Decreasing LDL cholesterol and blood pressure reduces the risk of cardiovascular events in populations of patients with diabetes [34]. In our study, lipid metabolism and blood pressure were improved in both groups of patients, although there was no statistically significant difference between the two groups. Thus, we concluded that RYGB can improve the patients' cardiovascular risk factors in both groups, which may in turn improve their life expectancy in the coming years.

There was no mortality and no need of reoperation in this study, and the overall morbidity of $9.5 \%$ was similar to large series in the literature, which reported an overall complication rate of 5-19\% [35]. A previous study reported a higher internal hernia rate in patients with a longer limb length [9]. The mesenteric and Petersen defects were closed to prevent these hernias in our hospital. A patient with severe malnutrition was cured through conservative treatment 1 year after surgery in this study. Also there were reports of 2 cases of severe malnutrition after mini-gastric bypass surgery $[19,36]$; unfortunately, 1 patient expired after all resuscitative measures were unsuccessful. Those case reports informed us that individualized treatment and long-term follow-up play a very important role in the whole treatment. 
This study had several limitations. First, the relatively minor change $(50 \mathrm{~cm}$ vs. $100 \mathrm{~cm}$ ) in the proportion of BPL may have limited the contribution to glucose control and weight loss. Secondly, the case number was relatively small and 3-year follow-up was not long enough to determine late complications and maintenance of diabetes remission. Last and most important, we failed to take into consideration the $\mathrm{CL}$, which could be a determinant factor for malabsorption.

\section{Conclusions}

RYGB is an effective procedure for the treatment of T2DM. With the total bypass ( $\mathrm{AL}+\mathrm{BPL}$ ) lengths consistent, lengthening of the BPL from 30 to $100 \mathrm{~cm}$ did not affect post-RYGB glucose control and weight loss.

\section{Acknowledgments}

The study was sponsored and funded by National Key Clinical Specialty Military Construction Project.

\section{Conflict of interest}

The authors declare no conflict of interest.

\section{References}

1. Welbourn R, Pournaras DJ, Dixon J, et al. Bariatric Surgery Worldwide: Baseline Demographic Description and One-Year Outcomes from the Second IFSO Global Registry Report 20132015. Obes Surg 2018; 28: 313-22.

2. Madan AK, Harper JL, Tichansky DS. Techniques of laparoscopic gastric bypass: on-line survey of American Society for Bariatric Surgery practicing surgeons, Surg Obes Relat Dis 2008; 4: 166-72.

3. Brolin RE, Kenler HA, Gorman JH, et al. Long-limb gastric bypass in the superobese. A prospective randomized study. Ann Surg 1992; 215: 387-95.

4. Brolin RE, LaMarca LB, Kenler HA, et al. Malabsorptive gastric bypass in patients with superobesity. J Gastrointest Surg 2002; 6: 195-203

5. Choban PS, Flancbaum L. The effect of Roux limb lengths on outcome after Roux-en-Y gastric bypass: a prospective, randomized clinical trial, Obes Surg 2002; 12: 540-5.

6. Feng JJ, Gagner M, Pomp A, et al. Effect of standard vs extended Roux limb length on weight loss outcomes after laparoscopic Roux-en-Y gastric bypass. Surg Endosc 2003; 17: 1055-60.

7. Ciovica R, Takata M, Vittinghoff E, et al. The impact of roux limb length on weight loss after gastric bypass. Obes Surg 2008; 18: 5-10.

8. Gleysteen JJ. Five-year outcome with gastric bypass: Roux limb length makes a difference. Surg Obes Relat Dis 2009; 5: 242-7.
9. Inabnet WB, Quinn T, Gagner M, et al. Laparoscopic Roux-en-Y gastric bypass in patients with $\mathrm{BMI}<50$ : a prospective randomized trial comparing short and long limb lengths. Obes Surg 2005; 15: 51-7.

10. Lee S, Sahagian KG, Schriver JP. Relationship between varying Roux limb lengths and weight loss in gastric bypass. Curr Surg 2006; 63: 259-63.

11. MacLean LD, Rhode BM, Nohr CW. Long- or short-limb gastric bypass? J Gastrointest Surg 2001; 5: 525-30.

12. Pinheiro JS, Schiavon CA, Pereira PB, et al. Long-long limb Rouxen-Y gastric bypass is more efficacious in treatment of type 2 diabetes and lipid disorders in super-obese patients. Surg Obes Relat Dis 2008; 4: 521-5.

13. Freeman JB, Kotlarewsky M, Phoenix C. Weight loss after extended gastric bypass. Obes Surg 1997; 7: 337-44.

14. Sarhan M, Choi JJ, Al Sawwaf M, et al. Is weight loss better sustained with long-limb gastric bypass in the super-obese? Obes Surg 2011; 21: 1337-43.

15. Valezi AC, Marson AC, Merguizo RA, et al. Roux-en-Y gastric bypass: limb length and weight loss. Arq Bras Cir Dig 2014; 27 Suppl 1: 56-8.

16. Darabi S, Pazouki A, Hosseini-Baharanchi FS, et al. The role of alimentary and biliopancreatic limb length in outcomes of Roux-en-Y gastric bypass. Videosurgery Miniinv 2020; 15: 290-7.

17. Kaska L, Kobiela J, Proczko M, et al. Does the length of the biliary limb influence medium-term laboratory remission of type 2 diabetes mellitus after Roux-en-Y gastric bypass in morbidly obese patients? Videosurgery Miniinv 2014; 9: 31-9.

18. Nelson WK, Fatima J, Houghton SG, et al. The malabsorptive very, very long limb Roux-en-Y gastric bypass for super obesity: results in 257 patients. Surgery 2006; 140: 517-22.

19. Motamedi MAK, Barzin M, Ebrahimi M, et al. Severe fatal protein malnutrition and liver failure in a morbidly obese patient after mini-gastric bypass surgery: case report. Int J Surg Case Rep 2017; 33: 71-4.

20. Elder KA, Wolfe BM. Bariatric surgery: a review of procedures and outcomes. Gastroenterology 2007; 132: 2253-71.

21. American Diabetes A, 2. Classification and Diagnosis of Diabetes, Diabetes Care 2017; 40 (Suppl 1): S11-24.

22. Kashyap SR, Bhatt DL, Schauer PR, et al. Bariatric surgery vs. advanced practice medical management in the treatment of type 2 diabetes mellitus: rationale and design of the Surgical Therapy And Medications Potentially Eradicate Diabetes Efficiently trial (STAMPEDE). Diabetes Obes Metab 2010; 12: 452-4.

23. Thaler JP, Cummings DE. Minireview: hormonal and metabolic mechanisms of diabetes remission after gastrointestinal surgery. Endocrinology 2009; 150: 2518-25.

24. Rubino F, Schauer PR, Kaplan LM, et al. Metabolic surgery to treat type 2 diabetes: clinical outcomes and mechanisms of action. Annu Rev Med 2010; 61: 393-411.

25. Rubino F, Forgione A, Cummings DE, et al. The mechanism of diabetes control after gastrointestinal bypass surgery reveals a role of the proximal small intestine in the pathophysiology of type 2 diabetes. Ann Surg 2006; 244: 741-9.

26. Dogan K, Homan J, Aarts EO, et al. A short or a long Roux limb in gastric bypass surgery: does it matter? Surg Endosc 2017; 31: 1882-90. 
27. Mahawar KK, Kumar P, Parmar C, et al. Small bowel limb lengths and Roux-en-Y gastric bypass: a systematic review. Obes Surg 2016; 26: 660-71.

28. Dallegrave Marchesini JC. End-to-side duodeno-jejunostomy with half-and-half biliopancreatic limb for the treatment of type 2 diabetes: a proposal for a simpler technique. Obes Surg 2007; 17: 138-9.

29. Stefanidis D, Kuwada TS, Gersin KS. The importance of the length of the limbs for gastric bypass patients: an evidence-based review. Obes Surg 2011; 21: 119-24.

30. Elliott JA, le Roux CW. How long should we make the biliopancreatic limb during Roux-en-Y gastric bypass? Surg Obes Relat Dis 2015; 11: 1246-7.

31. Kao YH, Lo CH, Huang CK. Relationship of bypassed limb length and remission of type 2 diabetes mellitus after Roux-en-Y gastric bypass. Surg Obes Relat Dis 2012; 8: e82-4.

32. Nergaard BJ, Leifsson BG, Hedenbro J, et al. Gastric bypass with long alimentary limb or long pancreato-biliary limb: long-term results on weight loss, resolution of co-morbidities and metabolic parameters. Obes Surg 2014; 24: 1595-602.

33. Alberti KG, Eckel RH, Grundy SM, et al. Harmonizing the metabolic syndrome: a joint interim statement of the International Diabetes Federation Task Force on Epidemiology and Prevention; National Heart, Lung, and Blood Institute; American Heart Association; World Heart Federation; International Atherosclerosis Society; and International Association for the Study of Obesity. Circulation 2009; 120: 1640-5.

34. American Diabetes A, 9. Cardiovascular Disease and Risk Management: Standards of Medical Care in Diabetes-2018. Diabetes Care 2018; 41 (Suppl 1): S86-104.

35. Lancaster RT, Hutter MM. Bands and bypasses: 30-day morbidity and mortality of bariatric surgical procedures as assessed by prospective, multi-center, risk-adjusted ACS-NSQIP data. Surg Endosc 2008; 22: 2554-63.

36. Dang H, Arias E, Szomstein S, et al. Laparoscopic conversion of distal mini-gastric bypass to proximal Roux-en-Y gastric bypass for malnutrition: case report and review of the literature. Surg Obes Relat Dis 2009; 5: 383-6.

Received: 19.07.2020, accepted: 12.09.2020. 